\title{
NONLINEAR BULGING FACTORS FOR LONGITUDINAL AND CIRCUMFERENTIAL CRACKS IN CYLINDRICAL SHELLS SUBJECTED TO COMBINED LOADS
}

\author{
Richard D. Young, ${ }^{*}$ Cheryl A. Rose, ${ }^{*}$ and James H. Starnes, Jr. ${ }^{\dagger}$ \\ NASA Langley Research Center \\ Hampton, Virginia 23681-001
}

\begin{abstract}
$\underline{\text { Abstract }}$
Results of a geometrically nonlinear finite element parametric study to determine curvature correction factors or "bulging factors" that account for increased crack-tip stresses due to curvature for longitudinal and circumferential cracks in unstiffened cylindrical shells subjected to combined loads are presented. Nondimensional parameters varied in the study include the shell curvature parameter, $\lambda$, which is a function of the shell radius, the shell wall thickness, and the crack length; a pressure loading parameter, $\eta$, which is a function of the shell geometry, material properties and the applied internal pressure; and a biaxial loading parameter, $\chi$, which is the ratio of the farfield axial stress to the farfield circumferential stress. The major results are presented in the form of contour plots of the bulging factor as a function of these three nondimensional parameters. These plots identify the ranges of the shell curvature and loading parameters for which the effects of geometric nonlinearity are significant, and show the effect of the biaxial loads on the value of the bulging factor. Simple empirical expressions for the bulging factor are then derived from the numerical results and are shown to predict accurately the nonlinear response of shells with longitudinal and circumferential cracks.
\end{abstract}

\section{Introduction}

The fail-safe design philosophy applied to transport aircraft fuselage structure requires that these structures retain adequate structural integrity in the presence of discrete source damage or fatigue cracks. Two types of damage most frequently associated with the structural integrity of fuselage structures are longitudinal cracks subjected to the circumferential stresses resulting from the internal pressure loads, and circumferential cracks subjected to stresses resulting from the bending and shearing of the fuselage induced by normal flight loads. The analysis of these fuselage cracks is complex due to the geometric coupling of bending and stretching deformations

\footnotetext{
*Aerospace Engineer, Mechanics and Durability Branch. Member, AIAA.

${ }^{\dagger}$ Chief Engineer, Structures and Materials Competency. Fellow, AIAA.

Copyright $(92000$ by the American Institute of Aeronautics and Astronautics,

Inc. No copyright is asserted in the United States under Title 17, U. S. Code. The

U. S. Government has a royalty-free license to exercise all rights under the copy-

right claimed herein for Governmental Purposes. All other rights are reserved by

the copyright owner.
}

in a shell structure, the geometrically nonlinear stiffening effect of membrane tensile stresses that are aligned with the crack faces, and the interaction of the crack with the surrounding structure (frames, stiffeners, and tear straps). To make the residual strength analyses of fuselage structure tractable in the design phase, current residual strength analyses and damage tolerant design practice rely primarily on geometrically linear analyses and fracture analyses based on linear elastic fracture mechanics. Linear elastic fracture mechanics suggests that the crack-tip stress intensity factor is an indicator of the likelihood of fracture. The conventional engineering approach used in design practice is to predict the crack-tip stress intensity factors for a crack in a fuselage shell by applying a so-called "bulging factor," in combination with additional design factors that account for stiffener elements, to the stress intensity factor for a flat plate subjected to similar loading conditions.

The bulging factor accounts for the fundamental difference in behavior of a crack in a curved shell compared to the behavior of a crack in a flat plate. In a cracked shell, the local region around the crack deforms out-of-plane as a result of the curvature induced coupling between the membrane and bending displacements, and the internal pressure, where, in a plate, the crack deforms in plane. These out-of-plane displacements in the neighborhood of a crack in a shell increase the crack opening and crack-tip stress intensity compared to those of a cracked plate with the same crack geometry. The bulging factor amplifies the flat-plate stress intensity factor and is defined as the ratio of the stress intensity factor in a cracked shell to the stress intensity factor in a cracked plate.

Many studies have been conducted to characterize bulging cracks, and both analytical ${ }^{1-8}$ and empirical formulas ${ }^{9-14}$ for the bulging factor have been developed. Analytical expressions for the bulging factor in shells were first developed by Folias using a formulation based on linear shallow shell theory. ${ }^{1-4}$ Folias' expressions depend on the shell curvature parameter, $\lambda$, where, for an isotropic shell, $\lambda$ is defined as:

$$
\lambda=\frac{a}{\sqrt{R} t} \sqrt[4]{12\left(1-v^{2}\right)}
$$


and:

$$
\begin{aligned}
v & =\text { Poisson's ratio } \\
a & =\text { half crack length } \\
R & =\text { radius of the shell } \\
t & =\text { thickness of the shell }
\end{aligned}
$$

Folias' original solutions are only valid for very small values of $\lambda$. The range of application of his solutions was later extended ${ }^{5-8}$ to larger values of $\lambda(\lambda=8$ for a longitudinal crack, $\lambda=10$ for a circumferential crack), by solving numerically the integral equations associated with the problem.

The analytical bulging factors tend to overestimate the physical bulging effect, unless the cracks are very short, or the applied load is very small, so that geometric nonlinear effects are not significant. The error introduced by the linearization of the shell equations has been explained by Riks, et al., ${ }^{15}$ and is a result of the tensile membrane stresses that develop along the crack edges as the crack bulges. These tensile stresses increase the resistance to additional crack bulging and crack opening. This nonlinear coupling between the bulging deformations and the membrane tensile stresses is not predicted by a linear analysis. Recently, some empirical formulas, which attempt to account for the nonlinear character of the bulging response, have been developed for determining bulging factors in shells with longitudinal cracks. ${ }^{11-}$ 14 These empirical formulas were developed for specific materials, geometries and loading conditions, and thus, the formulas are valid for limited applications.

A more general investigation of the geometrically nonlinear response of pressurized cylindrical shells with longitudinal cracks was conducted by Budiman and Lagace. ${ }^{16,17}$ Using the Donnell-Mushtari-Vlasov nonlinear shallow shell theory, ${ }^{18}$ Budiman and Lagace postulated that the nonlinear response is dependent on two nondimensional parameters: the shell curvature parameter, $\lambda$, which appears in the linear theory, and is defined in Eq. (1); and a loading parameter, $\eta$, which depends on the applied internal pressure, material properties, and shell geometry. From the nondimensional form of the governing equations, Budiman and Lagace ${ }^{16,17}$ defined $\eta$ as

$$
\eta_{\text {Budiman }}=\left(\frac{p R}{t}\right) \frac{1}{E} \frac{1}{\left(\frac{t}{R}\right)}
$$

where:

$$
\begin{aligned}
E & =\text { Young' } s \text { modulus } \\
p & =\text { internal pressure }
\end{aligned}
$$

and the remaining variables are as defined in Eq. (1). Budiman and Lagace stated that $\eta$ is a measure of the "driving force" of the nonlinearity. Budiman and
Lagace assessed the applicability of these parameters to the nonlinear response of pressurized cylindrical shells with longitudinal cracks by conducting nonlinear analyses of different cylindrical configurations using the STAGS finite element code. ${ }^{19}$ The results of the analyses showed that the two parameters are able to characterize the response of cylindrical shells with longitudinal cracks, subjected to internal pressure loading. Budiman ${ }^{17}$ also suggested, after obtaining the same two parameters from the nondimensional form of Sanders' nonlinear shell equations, that $\lambda$ and $\eta$ can be used to characterize the response of cylindrical shells when the crack is oriented in the circumferential direction, although Budiman did not confirm this hypothesis. Results of recent geometrically nonlinear finite element analyses presented by the present authors ${ }^{20}$ demonstrated that $\lambda$ and $\eta$ can also be used to characterize the geometrically nonlinear response of any pressurized cylindrical shell with a circumferential crack. Furthermore, the results presented in Ref. 20 show that the magnitude of the bulging factor for both longitudinal and circumferential cracks is affected by the shell geometry and the shell loading, and that for many shell geometries and load magnitudes the bulging factor is strongly influenced by the geometrically nonlinear response of a pressurized thin shell. The local response of the shell in the neighborhood of the crack is dominated by linear bending or nonlinear membrane response characteristics depending on the values of the shell radius, the shell thickness, the crack length, and the magnitude of the applied internal pressure load. When the internal pressure load is small, the local behavior of the shell is dominated by the linear bending response, and the bulging factor is a function of the shell curvature parameter, $\lambda$, only. For higher values of the internal pressure load, the response is dominated by membrane tension effects which reduce the bulging factor with increasing $\eta$. It was also shown in Ref. 20 that, in the nonlinear region of the response, the bulging factor can be uniquely related to the ratio of $\lambda / \eta$, where the loading parameter, $\eta$, is defined as:

$$
\eta=\sqrt{\frac{\sigma_{y}}{E}} \frac{R}{t}\left(\sqrt[4]{12\left(1-v^{2}\right)}\right)
$$

where $\sigma_{y}$ is the farfield circumferential stress.

The studies described in the previous paragraph, and the majority of the studies presented in the literature are for the case of a shell with a crack subjected to internal pressure loads and the axial load associated with the bulkhead loads, without consideration of the effect of additional axial mechanical loads. Recent studies have shown, however, that for shells with longitudinal cracks, the ratio of the farfield axial stress to the farfield circumferential stress, or biaxial loading parameter 
$\chi=\sigma_{x} / \sigma_{y}$, can have a significant effect on the bulging deformations and the bulging factors. ${ }^{11,21}$ Consequently, formulas developed for the loading condition of internal pressure only, $\chi=0.5$, may lead to unsafe designs if their predictions are non-conservative, or conversely, to excessive structural weight if they are overly conservative.

The present paper has several objectives. The first objective is to present the results of a comprehensive geometrically nonlinear numerical parametric study of the response of aluminum shells with centrally located longitudinal and circumferential cracks subjected to combined internal pressure and mechanical loads. Geometric parameters varied in the parametric study include the shell radius, the shell wall thickness, the crack length, and the crack orientation. The second objective is to assess the dependence of the nonlinear response of shells with longitudinal and circumferential cracks on the geometric parameter, $\lambda$, the pressure loading parameter, $\eta$, and the biaxial loading parameter, $\chi$. The final objective is to develop simple expressions for determining the bulging factors, that can be used easily in a design environment, for longitudinal and circumferential cracks in cylindrical shells subjected to combined internal pressure and mechanical loads.

\section{Strain-Energy Release Rate and Bulging Factor}

Linear elasticity theory predicts a stress singularity at the tips of cracks, and the strength of the crack-tip stress field singularity is represented by the stress intensity factor, $K$. For a flat plate with a central crack subjected to uniaxial tension perpendicular to the crack direction, the stress intensity factor for the plate, $K_{p}$, is defined as

$$
K_{p}=\sigma \sqrt{\pi a} f(W)
$$

where $\sigma$ is the in-plane remote stress acting perpendicular to the crack line, and $f(W)$ is a function to account for finite width effects. For a flat plate, or in cases when the linear shell equations apply, the crack-tip stress field and the stress intensity factor are proportional to the loads, and the stress intensity factors can be related to the strain-energy release rate. ${ }^{22}$ For a flat plate with a central crack subjected to uniaxial tension perpendicular to the crack direction, the relationship between the stress intensity factor and the strain-energy release rate, $G$, has the form:

$$
G=\frac{K_{p}^{2}}{E}
$$

where $E$ is Young's modulus. When geometrically nonlinear effects are present, the stress field, and hence, the stress intensity factor, are not linear functions of the applied load, and the stress intensity factor cannot be defined as in Eq. (4). To address this problem, an engineering approach is employed and the nonlinear stress intensity factor for the shell, $K_{s}$, is defined on the basis of Eq. (5). ${ }^{23}$ For the present study, the stress intensity factor $K_{s}$ is calculated from:

$$
K_{s}=\sqrt{E G}
$$

For the symmetric loading conditions considered in the present paper, $K_{s}$ defined by Eq. (6) is the total stress intensity factor, and is a combination of the symmetric membrane and bending stress intensity factors, $K_{I}$ and $k_{1}$, respectively. ${ }^{24}$ In the present paper, only the total stress intensity factor $K_{s}$ is considered. The stress intensity factor $K_{s}$ is related through a bulging factor to the stress intensity factor for the reference problem of a flat plate with a central crack subjected to uniaxial tension perpendicular to the crack direction. The bulging factor, $\beta$, is defined as the ratio of the stress intensity factor $K_{s}$ in a shell with a crack, to the stress intensity factor $K_{p}$ in a flat plate of the same material, thickness, crack length, and in-plane remote stress, $\sigma$, acting perpendicular to the crack line:

$$
\beta=\frac{K_{s}}{K_{p}}
$$

In the present study, the bulging factor for a cylindrical shell with a longitudinal crack or a circumferential crack is denoted as $\beta^{L}$ and $\beta^{C}$, respectively.

\section{Shell Geometry and Analysis Procedure}

\section{$\underline{\text { Shell Model }}$}

The geometry of a typical shell analyzed in the present study is defined in Fig. 1. The shell shown in Fig. 1 is a segment of an infinitely long cylindrical shell, with an infinite number of equal length longitudinal or circumferential cracks evenly distributed along the length of the shell to maintain the symmetry of the models. The shell is made of 2024-T3 aluminum alloy and has a radius, $R$, an axial length, $L_{a}$, a circumferential length, $L_{c}$, a wall thickness, $t$, and a crack length, $2 a$. The crack is centrally located and is oriented longitudinally (parallel to the x-axis) or circumferentially (parallel to the y-axis). The Young's modulus, $E$, for the aluminum alloy is equal to $10.35 \mathrm{msi}$ and Poisson's ratio, $v$, is equal to 0.3 . The loading condition for the shell consists of an applied internal pressure, $p$, which generates a circumferential stress reaction, $\sigma_{y}$, and an axial stress, $\sigma_{x}$, which is the sum of the stress from a bulkhead pressure load, $\sigma_{x p}$, and an applied mechanical load, $\sigma_{x m}$. Seven values of the biaxial loading parameter are considered: $\chi=\sigma_{x} / \sigma_{y}=0,0.25,0.5,1.0,1.5,3.0$, and 6.0. A biaxial loading parameter $\chi=0.5$ corresponds to the internal pressure only loading condition. 


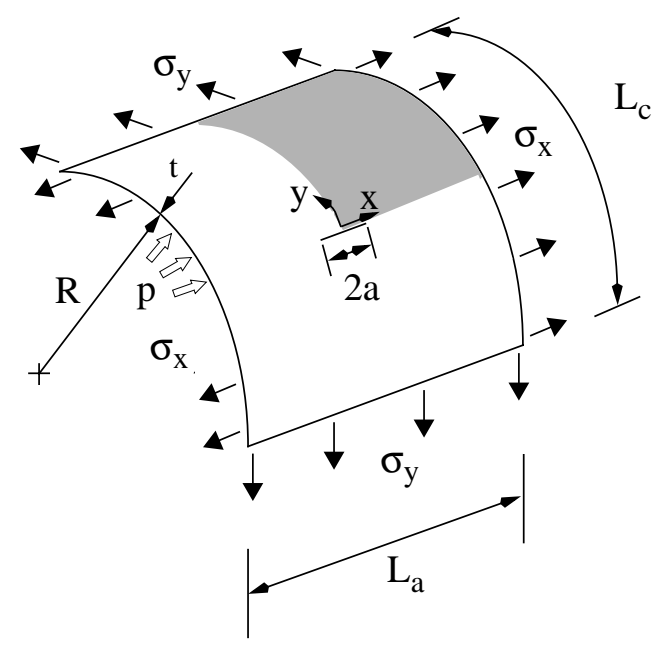

Figure 1. Shell Geometry.

Typical finite element models used to simulate the response of the cracked shells are shown in Fig. 2, for shells with two different crack orientations. Quarter symmetry was assumed, so only the shaded portion of the shell segment shown in Fig. 1 was modeled. To simplify model generation for the wide range of parameters considered, the model dimensions in the $x$ and $y$ directions, and the element dimensions were scaled by the crack length. This approach was used to reduce the effort required to model shells with different crack lengths, while maintaining reasonable consistency in the solution resolution in going from a mesh for a short crack to a mesh for a long crack. The dimensions of the models in the $x$ and $y$ directions were set equal to $24 a$ and $6 a$, respectively. These dimensions were chosen to reduce finite width and finite length effects to an acceptable level; that is, changing the edge conditions resulted in less than a 5\% change in the computed stress intensity factor. The longer dimension in the longitudinal direction was required to minimize edge effects for the cylindrical shell with a circumferential crack.

The shells were modeled using STAGS standard 410 quadrilateral shell elements, and 510 and 710 meshtransition elements, where needed. The elements are flat facet-type elements and are based on Kirchoff-Love shell theory and the nonlinear Lagrangian strain tensor. ${ }^{19,25}$ Each of the shell element nodes has six degrees of freedom, including three translational degrees of freedom, $u, v$, and $w$, and three rotational degrees of freedom, $r u, \quad r v$, and $r w$ about the axes $x, y$, and $z$, respectively (see Fig. 1). Symmetry boundary conditions were prescribed on the left $(x=0)$ and bottom $(y=0)$ edges of the model. Periodic boundary conditions were prescribed to approximate the physical boundary conditions on the top

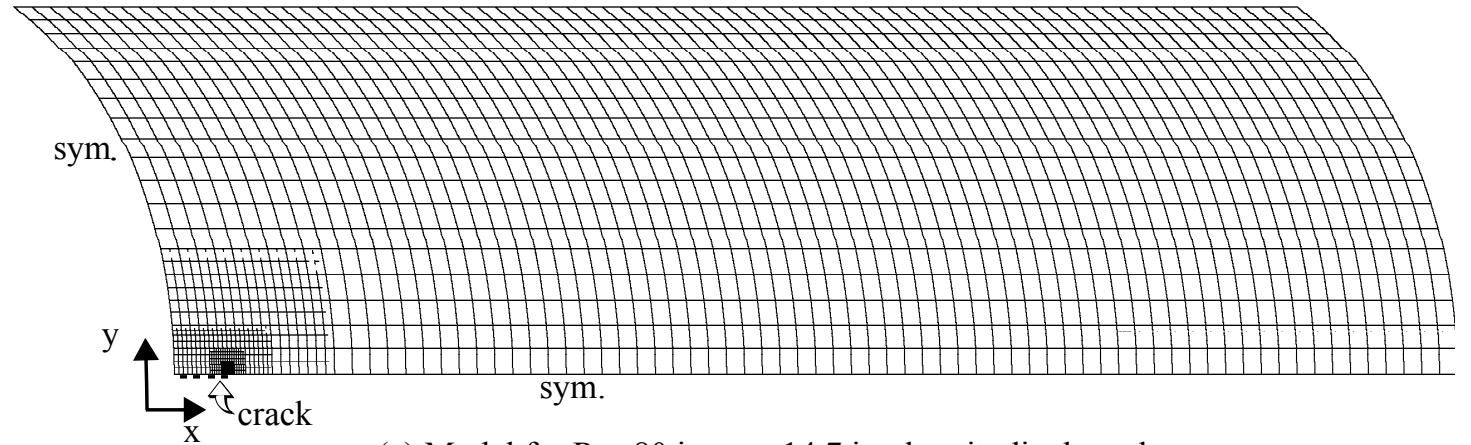

(a) Model for $\mathrm{R}=80$ in., $\mathrm{a}=14.7$ in., longitudinal crack

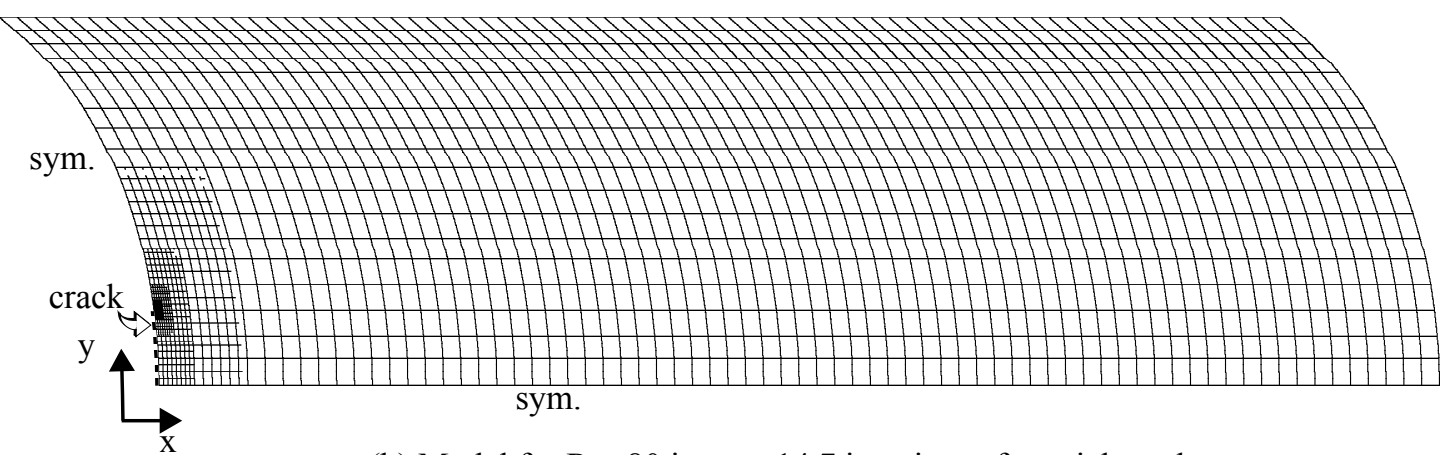

(b) Model for $\mathrm{R}=80$ in., $\mathrm{a}=14.7$ in., circumferential crack

Figure 2. Typical finite element models. 
$(y=6 a / R)$ and right edges $(x=24 a)$ of the model. The right edge of the model was also constrained to remain cylindrical throughout the loading process. Specifically, on the top edge of the model, the circumferential degree of freedom, $v$, and the rotational degrees of freedom, $r u$, and $r w$, were set equal to zero; and on the right edge of the model, the axial and radial degrees of freedom, $u$, and $w$, respectively, were constrained to be uniform, and the rotational degrees of freedom, $r v$, and $r w$, were set equal to zero. A symmetric crack with only one side of the crack modeled was defined along the bottom edge (longitudinal crack) or left edge (circumferential crack) of the model. The crack has a half crack length equal to $a$ and starts in the lower left corner of the model and extends to the right (longitudinal crack), or up (circumferential crack), as shown in Fig. 2. A fine mesh was required to represent the stress and deformation gradients near the crack tip. To eliminate the dependence of the results on mesh resolution, several analyses were conducted, with increasing mesh refinement in the cracktip region, until further refinement produced less than $1 \%$ change in the total stress intensity factor, $K_{s}$. The analyses converged using elements near the crack tip with edge lengths equal to $0.01 a$. Predictions of the flat-plate stress intensity factor using the converged mesh were within $1 \%$ of the predictions obtained using Eq. (4), with Irwin's finite width adjustment. ${ }^{22}$ The loading on the shell consisted of two parts. Internal pressure was simulated by applying a uniform lateral pressure to the shell wall and an axial tensile force to account for bulkhead pressure loads to the right edge of the shell, $\sigma_{x}=\left(\sigma_{y} / 2\right)$, with multi-point constraints to enforce a uniform edge displacement. The 'internal pressure only' load case is then represented by a biaxial loading parameter, $\chi=0.5$. The internal pressure plus mechanical load cases, $(\chi \neq 0.5)$, were simulated by applying an additional axial force to the right edge of the model.

\section{$\underline{\text { Nonlinear Analysis Procedure }}$}

The shell responses were predicted numerically using the STAGS (STructural Analysis of General Shells) nonlinear shell analysis code. ${ }^{19}$ STAGS is a finite element code for general-purpose analysis of shells of arbitrary shape and complexity. STAGS analysis capabilities include stress, stability, vibration and transient response analyses, with both material and geometric nonlinearities represented. The code uses both the modified and full Newton methods for its nonlinear solution algorithms, and accounts for large rotations in a shell by using a co-rotational algorithm at the element level. The Riks pseudo arc-length path following method ${ }^{26,27}$ is used to continue a solution past limit points in a nonlinear response. The strain-energy release rate is calcu- lated in STAGS, from a nonlinear equilibrium state, using the modified crack closure integral technique. ${ }^{28}$

\section{$\underline{\text { Results and Discussion }}$}

The geometrically nonlinear analysis results for unstiffened cylindrical shells with a longitudinal or circumferential crack are presented in this section. This section is separated into three parts. Each part corresponds to a phase in the nonlinear analysis approach that was used to assess the applicability of the nondimensional parameters $\lambda, \eta$ and $\chi$, for characterizing the nonlinear response of a shell with a crack, and to study the effect of these parameters on the nonlinear shell response. In the first part, the accuracy of the analysis model and the analysis procedure is assessed by comparing the STAGS linear predictions for the bulging factor with solutions in the literature that are based on linear shallow shell theory. The linear analyses were performed for 30 shells with a radius equal to $80 \mathrm{in}$., a shell wall thickness equal to $0.040 \mathrm{in}$., and half-crack lengths ranging from $0.49 \mathrm{in}$. to 14.76 in. In the second part, results of the analyses conducted for the specific shell configurations shown subsequently in Table 1 are presented to confirm the applicability of the nondimensional parameters $\lambda, \eta$ and $\chi$, for characterizing the nonlinear response of shells with longitudinal and circumferential cracks. The shell configurations shown in Table 1 represent a variation of the shell curvature parameter of $2.875 \leq \lambda \leq 11.50$. The third part presents results of a series of nonlinear analyses that were conducted to determine the bulging factors for a large range of the nondimensional parameters. Simple empirical expressions for the bulging factor are then derived from the numerical results and shown to predict accurately the nonlinear response of shells with longitudinal and circumferential cracks. In all of the analyses, the loading condition for the shell consisted of an applied internal pressure, $p$, which generates a circumferential stress reaction, $\sigma_{y}$, and an axial stress, $\sigma_{x}$, giving a biaxial loading parameter $\chi=\left(\sigma_{x} / \sigma_{y}\right)=0.0$, $0.25,0.50,1.0,1.5,3.0$, or 6.0 . The 'pressure only' case is given by $\chi=0.5$. Internal pressure was varied such that $0 \leq \eta \leq 3.0$, where in the present study the pressure loading parameter, $\eta$, is defined as:

$$
\eta=\sqrt{\frac{\sigma_{y}}{E}} \frac{R}{t}\left(\sqrt[4]{12\left(1-v^{2}\right)}\right)
$$

where $\sigma_{y}=p R / t$. All computations were performed using $E=10.35 \mathrm{msi}$ and $\mathrm{v}=0.3$.

\section{Linear Bulging Factors for Shells with Longitudinal and Circumferential Cracks}

In a linear analysis, the bulging factor depends only on the shell curvature parameter, $\lambda$. The linear bulging factors as a function of $\lambda$, computed using a linear 


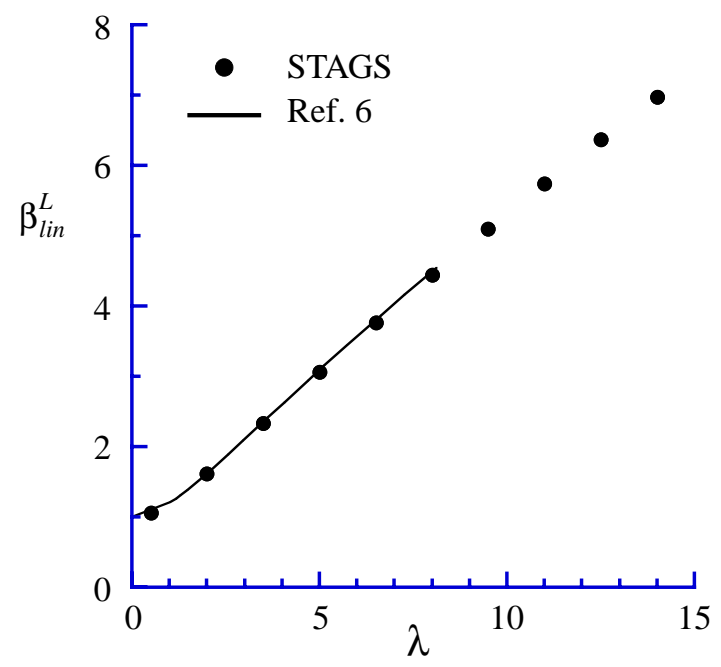

(a) Linear bulging factor for a longitudinal crack, $\beta_{\text {lin }}^{L}$

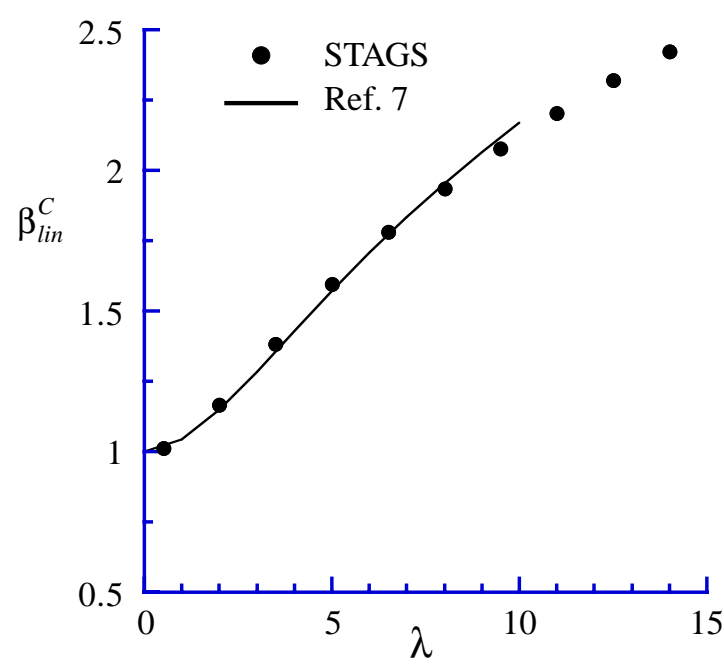

(b) Linear bulging factor for a circumferential crack, $\beta_{\text {lin }}^{C}$

Figure 3. Linear bulging factors versus $\lambda$, predicted using STAGS and the numerical solution of Erdogan and Kibler $^{6}$ (longitudinal crack) and Erdogan and Ratwani ${ }^{7}$ (circumferential crack).

STAGS analysis, are compared in Fig. 3 with bulging factors computed from numerical solutions that are based on linear shallow shell theory. The linear bulging factors for a shell with a longitudinal crack, $\beta_{l i n}^{L}$, and for a shell with a circumferential crack, $\beta_{l i n}^{C}$, are shown in Figs. $3 \mathrm{a}$ and $3 \mathrm{~b}$, respectively. The numerical solutions from the literature are available for values of $\lambda$ less than 8 for the case of a longitudinal crack, ${ }^{6}$ and for values of $\lambda$ less than 10 for the case of a circumferential crack. ${ }^{7}$ The predictions shown in Fig. 3 were obtained using a configuration with a radius, $R=80$ in., thickness, $t=$ 0.040 in., and with the half-crack length, $a$, varied to provide the range of $\lambda$ values shown. The agreement between the geometrically linear finite element predictions, shown by the symbols, and the numerical solutions presented in Refs. 6 and 7, shown by the solid lines, is excellent, indicating that the mesh refinement near the crack tip, and that the shell dimensions chosen for the models, are adequate.

Assessment of $\lambda, \eta$ and $\chi$ as Governing Parameters for the Nonlinear Response of Shells with Longitudinal and Circumferential Cracks

Following Budiman and Lagace, ${ }^{16}$ the second step in the analysis was to confirm the applicability of the shell curvature parameter, $\lambda$, the pressure loading parameter, $\eta$, and the biaxial loading parameter, $\chi$, for characterizing the nonlinear response of cylindrical shells with both longitudinal and circumferential cracks subjected to internal pressure and mechanical loads. Cylindrical shell configurations considered in this phase of the analysis are provided in Table 1. As shown in Table 1, sixteen different cylindrical shell configurations with values of $\lambda$ equal to $2.875,5.75,8.625$, and 11.50 were considered. For each value of $\lambda$ and $\chi$, nonlinear analyses were performed for two values of the shell radius, and shell wall thickness, with the half-crack length adjusted accordingly. The larger radius, $R=80 \mathrm{in}$., is representative of a narrow-body transport fuselage geometry, and the smaller radius, $R=20 \mathrm{in}$., is representative of a relatively large laboratory scale specimen.

Previous results presented by Budiman and Lagace $^{16}$ and Young et. $\mathrm{al}^{20}$ confirmed that the nondimensional parameters $\lambda$ and $\eta$ can be used to characterize the bulging response of cylindrical shells with longitudinal and circumferential cracks subjected to internal pressure and the associated bulkhead loads $(\chi=$ 0.5 ) by showing that for different cylindrical shell configurations with cracks the nonlinear bulging factor response collapses to a single curve for a constant value of $\lambda$, when plotted as a function of $\eta$. Similar results were obtained in the present analysis, for shells with longitudinal and circumferential cracks, for all values of the biaxial loading parameter that were considered.

Representative nonlinear analysis results for the bulging factor of a shell with a longitudinal crack, $\beta^{L}$, of the configurations outlined in Table 1 with values of $\lambda$ equal to 2.875 and 11.50 are shown in Figs. $4 \mathrm{a}$ and $4 \mathrm{~b}$, respectively, as a function of the pressure loading parameter, $\eta$, and the biaxial loading parameter, $\chi$. Curves are 
Table 1. Configurations considered to confirm the applicability of nondimensional parameters, $\lambda$ and $\eta$

\begin{tabular}{|c|c|c|c|c|c|}
\hline & & $\lambda=2.875$ & $\lambda=5.75$ & $\lambda=8.625$ & $\lambda=11.50$ \\
\hline \multirow[t]{2}{*}{$\mathrm{R}=20$ in. } & $\mathrm{t}=0.02 \mathrm{in}$. & $\mathrm{a}=1 \mathrm{in}$. & $\mathrm{a}=2 \mathrm{in}$. & $\mathrm{a}=3 \mathrm{in}$. & $\mathrm{a}=4 \mathrm{in}$. \\
\hline & $\mathrm{t}=0.08$ in. & $\mathrm{a}=2$ in. & $\mathrm{a}=4 \mathrm{in}$. & $a=6$ in. & $\mathrm{a}=8$ in. \\
\hline \multirow[t]{2}{*}{$\mathrm{R}=80$ in. } & $\mathrm{t}=0.02$ in. & $\mathrm{a}=2$ in. & $\mathrm{a}=4 \mathrm{in}$. & $\mathrm{a}=6 \mathrm{in}$. & $\mathrm{a}=8 \mathrm{in}$. \\
\hline & $\mathrm{t}=0.08$ in. & $\mathrm{a}=4 \mathrm{in}$. & $\mathrm{a}=8 \mathrm{in}$ & $\mathrm{a}=12 \mathrm{in}$. & $\mathrm{a}=16$ in. \\
\hline
\end{tabular}

shown for values of $\chi=0.0,0.5,1.5$, and 6.0. For each value of $\lambda$ in Table 1 , there are four cylindrical shell configurations. The results in Fig. 4 show that when the nonlinear bulging factors are presented as a function of $\eta$ and $\chi$, the response for the different shells with equal values of $\lambda$ collapses to a single curve for each value of the biaxial loading parameter. That is, each curve in Fig. 4, labeled with a constant value of $\chi$, corresponds to the bulging factor predictions for four different shell configurations. Similar results are obtained for the shell configurations in Table 1 with values of $\lambda$ equal to 5.75 and 8.625. Therefore, the results of the analyses confirm the use of the parameters $\lambda, \eta$ and $\chi$ to characterize the nonlinear response of shells with longitudinal cracks and subjected to internal pressure and axial loads. Furthermore, the results in Fig. 4 show that the bulging factor response is a nonlinear function of the pressure loading parameter, $\eta$, for all values of the biaxial loading parameter, $\chi$, and the shell curvature parameter, $\lambda$. For small values of $\eta(\eta<<1)$ the local shell response is predominantly a linear bending response, and the bulging factors

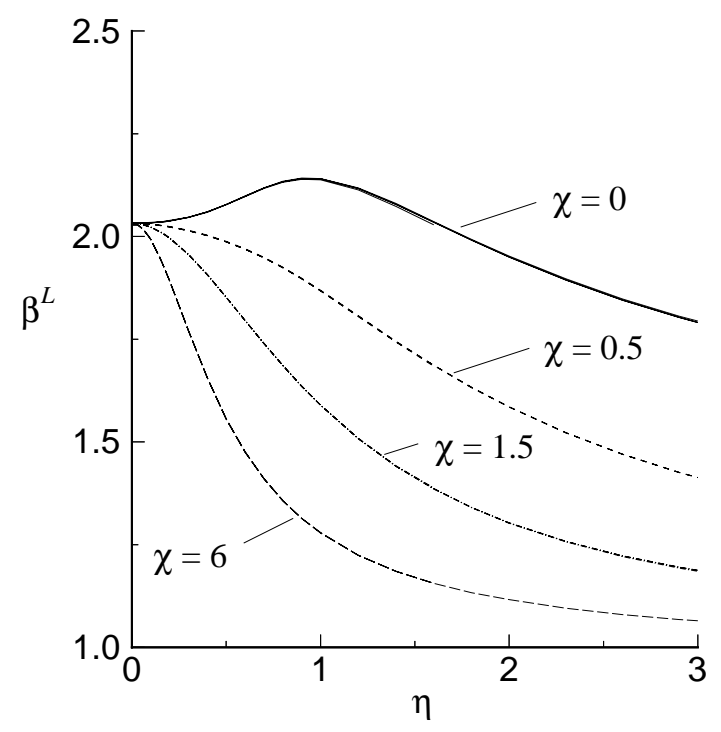

(a) $\lambda=2.875$ from the nonlinear analysis are independent of the pressure loading parameter, $\eta$, and the biaxial loading parameter, $\chi$, and therefore, depend only on $\lambda$. The linear response is indicated by the flat regions of the curves for values of $\eta$ near zero in Fig. 4. For larger values of $\eta$, the response transitions from a linear bending dominated response to a nonlinear response, where the bulging factor is dependent on the pressure loading parameter, $\eta$. The rate at which the response transitions from linear to nonlinear, and the nature of the initial nonlinear response is dependent upon both the biaxial loading parameter, $\chi$, and the shell curvature parameter, $\lambda$.

When a shell with a longitudinal crack is initially loaded by internal pressure, compressive axial membrane stresses develop along the edges of the crack, much like the compressive stresses that develop in regions near the edge of the crack in a center-cracked flat plate subjected to a tension load. Remote axial stresses applied to the shell influence the local axial membrane stresses along the edge of the crack and the bulging deformations. When the biaxial loading parameter, $\chi$, is

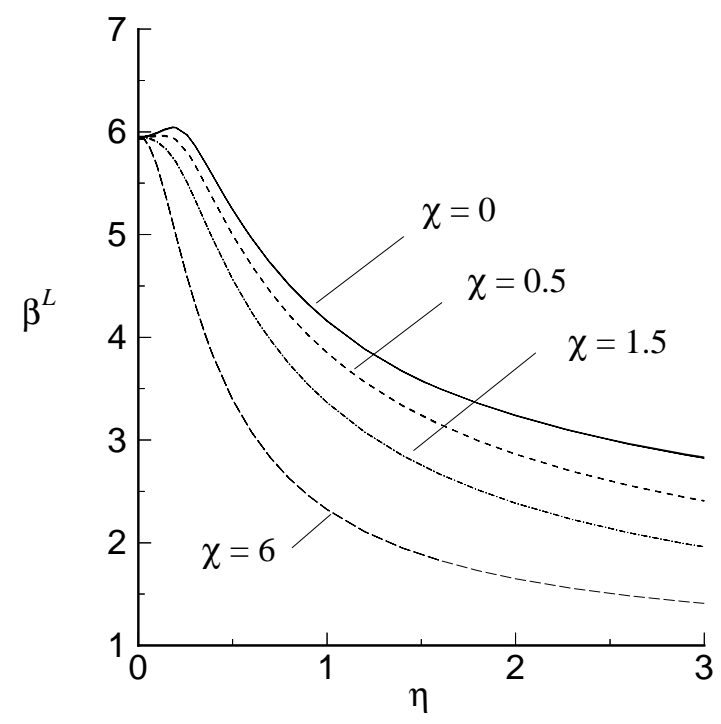

(b) $\lambda=11.50$

Figure 4. Dependence of the bulging factor for a longitudinal crack, $\beta^{L}$, on the pressure loading parameter, $\eta$, and the biaxial loading parameter, $\chi$, for two values of the shell curvature parameter, $\lambda$. 
equal to zero, the remote axial stress is zero, and the axial membrane stresses along the edge of the crack are compressive when $\eta$ is small. As $\eta$ is increased, the linear bending deformations become sufficiently large for the axial compressive stresses along the crack to couple with the out-of-plane bending deformations, causing a destabilizing effect, and the bulging factor initially increases with increasing $\eta$. As $\eta$ is increased further, the bulging deformations become large enough for tensile axial membrane stresses to develop along the crack edges. These tensile stresses increase the resistance to additional crack bulging and crack opening, and the bulging factor decreases with increasing values of $\eta$. For larger values of $\chi,(\chi=0.5,1.5,6.0$ in Fig. 4$)$ the local shell response is predominantly a nonlinear membrane dominated response, where the bulging factor decreases with increasing pressure, from the initiation of pressure loading. As $\chi$ is increased, the axial membrane stresses along the edge of the crack become tensile at increasingly smaller values of the pressure loading parameter, $\eta$. As described previously, these tensile stresses suppress the bulging deformations and the bulging factors are smaller for larger values of $\chi$. For configurations with large values of $\lambda$ (longer crack lengths), the response is more nonlinear than for shells with small values of $\lambda$ (shorter crack lengths) as indicated by the steeper slopes of the curves for $\lambda=11.50$ in Fig. $4 \mathrm{~b}$ as compared to the slope of the curves for $\lambda=2.875$ in Fig. $4 \mathrm{a}$.

The applicability of the shell curvature parameter, $\lambda$, and the loading parameters $\eta$ and $\chi$, for characterizing the nonlinear response of circumferentially crack cylindrical shells subjected to internal pressure and axial loads

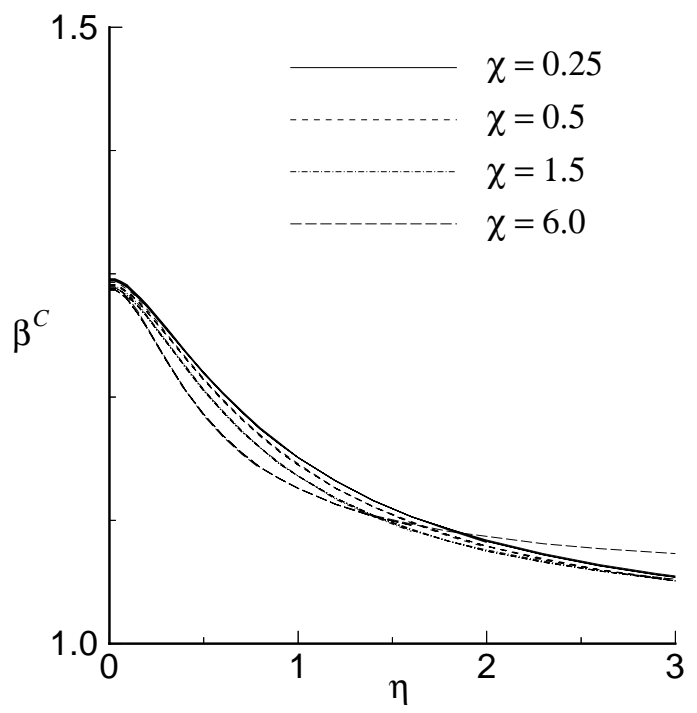

(a) $\lambda=2.875$ is also assessed. Nonlinear analyses were conducted for the same shell configurations as for the longitudinal crack (Table 1), and for values of the biaxial loading parameter equal to $0.25,0.50,1.0,1.5,3.0$, and 6.0. A biaxial loading parameter equal to zero was not considered for the cylinders with circumferential cracks, since this corresponds to the case of zero axial load and no stress intensity at the crack tip.

Results of the nonlinear analyses for configurations with $\lambda$ equal to 2.875 and 11.50 , and values of the biaxial loading parameter $\chi=0.25,0.50,1.5$, and 6.0 are summarized in Fig. 5. For very small values of $\eta$, the nonlinear bulging factor results are equal to the linear bulging factor. The results in Figs. $5 \mathrm{a}$ and $5 \mathrm{~b}$ for $\lambda$ equal to 2.875 and 11.50 , respectively, show that when the nonlinear bulging factors for circumferential cracks, $\beta^{C}$, are presented as a function of $\eta$, for each value of $\chi$, the responses for four different shells with the same value of $\lambda$ nominally collapse to a single curve. The bulging factor predictions for the second configuration in Table $1, R=$ 20 in., and $=0.08$ in., $(R / t=250)$ are slightly higher than the predictions for the other configurations because of mesh transition effects that are dependent on $R / t$. The results in Fig. 5 also show that the bulging factors for circumferential cracks are only mildly dependent on the biaxial loading parameter, and the maximum percentage difference between the bulging factor predictions for $\chi=0.25$ compared to the predictions for $\chi=6.0$ is $10 \%$. Similar results are obtained for shell configurations with values of $\lambda$ equal to 5.75 and 8.625. Therefore, the results of the analyses suggest that only the parameters $\lambda$ and $\eta$ are required to characterize the nonlinear response

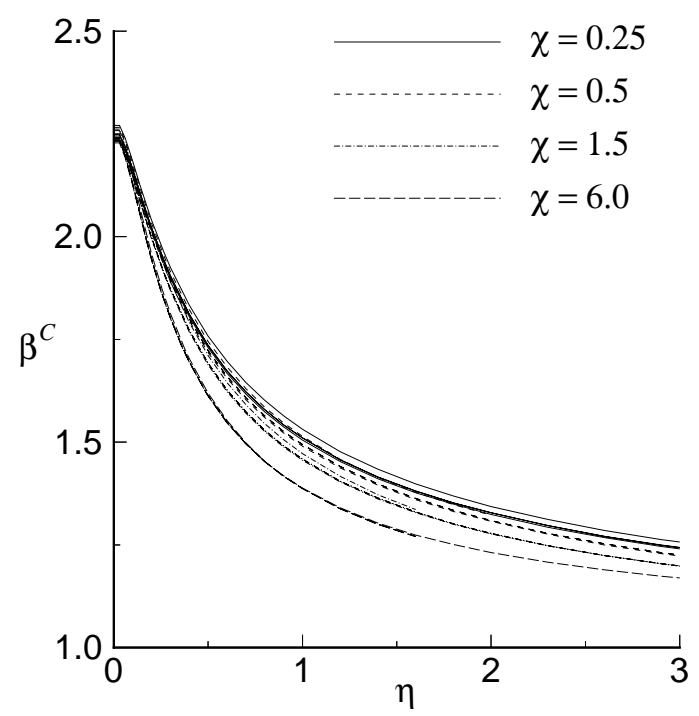

(b) $\lambda=11.50$

Figure 5. Dependence of the bulging factor for a circumferential crack, $\beta^{C}$, on the pressure loading parameter, $\eta$, and the biaxial loading parameter, $\chi$, for two values of the shell curvature parameter, $\lambda$. 
of shells with circumferential cracks and subjected to internal pressure and axial loads. Furthermore, for a given shell and crack length (given $\lambda$ ), geometric nonlinearities affect the bulging response, and become significant for very small values of the loading parameter, $\eta$, as indicated by the immediate decline in the magnitude of the bulging factor for values of $\eta>0$. For a given shell, the longer the crack length (larger $\lambda$ ), the more nonlinear the response, as indicated by the steeper slopes of the curves for shells with $\lambda=11.50$ compared to the curves for shells with $\lambda=2.875$.

\section{Bulging Factors for a Large Range of the Nondimen- sional Parameters}

The results presented in the previous section show that in an unstiffened cylindrical shell the bulging effect for a circumferential crack can be characterized adequately by the two nondimensional parameters, $\lambda$ and $\eta$, while the bulging effect for a longitudinal crack requires three nondimensional parameters, $\lambda, \eta$ and $\chi$, to characterize the response. To develop expressions for bulging factors for circumferential and longitudinal cracks in terms of these nondimensional parameters, a series of nonlinear analyses were conducted to determine the bulging factors for a large range of the nondimensional parameters. The analyses were conducted using shell configurations with $R=80.0$ in., $t=0.04$ in., and halfcrack lengths to provide values of $0.5 \leq \lambda \leq 15$ in increments of 0.5 . The internal pressure was varied such that $0 \leq \eta \leq 3.0$. For the circumferential crack, a single value of the biaxial loading parameter, $\chi=0.5$, was considered. For the longitudinal crack, values of the biaxial loading parameter of $\chi=0.0,0.25,0.5,1.0,1.5,3.0$ and 6.0 were considered. In the following figures, the bulging factor results from the analyses are plotted in terms of the nondimensional parameters, and the trends in the results are used to suggest functional forms which can simulate the observed response. Contour plots of the bulging factor results for a specific value of $\chi$ are presented as a function of the shell curvature parameter, $\lambda$, and the pressure loading parameter, $\eta$, to establish the functional dependence on $\lambda$ and $\eta$. For the longitudinal crack case, the variation of the functional dependence on $\lambda$ and $\eta$ for different values of the biaxial loading ratio, $\chi$, is used to suggest the functional dependence on $\chi$. Results are presented first for the longitudinal crack, and then for the circumferential crack.

Longitudinal Crack. The bulging factors from STAGS analyses of cylindrical shells with longitudinal cracks, $\beta^{L}$, as a function of the shell curvature parameter, $\lambda$, and the pressure loading parameter, $\eta$, are presented as contour plots in Fig. 6. Bulging factors for $\chi=0.0,0.5$, 1.5, and 6.0, are shown in Figs. $6 a, 6 b, 6 c$, and $6 \mathrm{~d}$, respectively. The solid lines in the figure are contour lines, or lines through points with a common value of the bulging factor. There are some general trends indicated by the contour plots. For a given value of $\chi$, the bulging factors monotonically increase with increasing values of $\lambda$, and generally decrease with increasing values of $\eta$. In each contour plot, the bulging factor for very small values of $\eta$, i.e., for locations near the $\lambda$-axis, corresponds to the linear bulging factor, $\beta_{\text {lin }}^{L}$, shown previously in Fig. 3a, and does not vary with changes in $\chi$. For small values of $\eta$, the contour lines are nearly perpendicular to the $\lambda$-axis, indicating that the bulging factor for small values of $\eta$ is primarily a function of $\lambda$ only and can be approximated by $\beta_{\text {lin }}^{L}$. The unshaded areas of the contour plots in Fig. 6 indicate the linear region of the response where the difference between $\beta^{L}$ and $\beta_{\text {lin }}^{L}$ is less than $10 \%$. For higher values of $\eta$, the linear bending deformations become sufficiently large and cause nonlinear membrane stiffening. The bulging factors decrease with increasing $\eta$, and the contour lines bend to the right and asymptotically approach lines which extend radially from the origin. The shaded areas of the contour plots in Fig. 6 indicate the nonlinear region of the response where the difference between $\beta^{L}$ and $\beta_{\text {lin }}^{L}$ is greater than $10 \%$. The largest differences between $\beta^{L}$ and $\beta_{\text {lin }}^{L}$ occur when $\lambda$ and $\eta$ are both large, where $\beta_{\text {lin }}^{L}$ overpredicts $\beta^{L}$ by $45 \%$ when $\chi=0$, and by $400 \%$ when $\chi=6$. Comparison of the contour plots for different values of $\chi$ indicates that increasing the biaxial loading parameter promotes tensile membrane behavior, causing the contour lines to bend to the right at lower values of $\eta$, thus reducing the size of the linear response region. A simple expression for representing the bulging factor behavior shown in Fig. 6, that can be easily used in a design environment, is obtained by characterizing the linear and nonlinear regions of the response separately.

The linear region of the response is accurately described by the linear bulging factor for a longitudinal crack, $\beta_{\text {lin }}^{L}$, shown previously in Fig. 3a. A simple expression for the linear bulging factor is obtained by examining the behavior of the numerical data and determining that the data can be approximated closely by the function

$$
\beta_{\text {lin }}^{L}=\sqrt{1+(0.5) \lambda^{1.725}}
$$

An expression for estimating the bulging factor in the nonlinear region of the response, $\beta_{n l}^{L}$, is obtained by utilizing the fact that the contour lines of the bulging factor asymptotically approach radial lines through the origin. For a given value of $\chi$, the value of the bulging factor can be uniquely related to the slope of the radial line, i.e., $\lambda / \eta$, which is approached asymptotically by a contour line. To obtain an expression which relates the nonlinear bulging factor to the ratio $\lambda / \eta$ and the biaxial loading ratio, $\chi$, the relationship between the nonlinear 


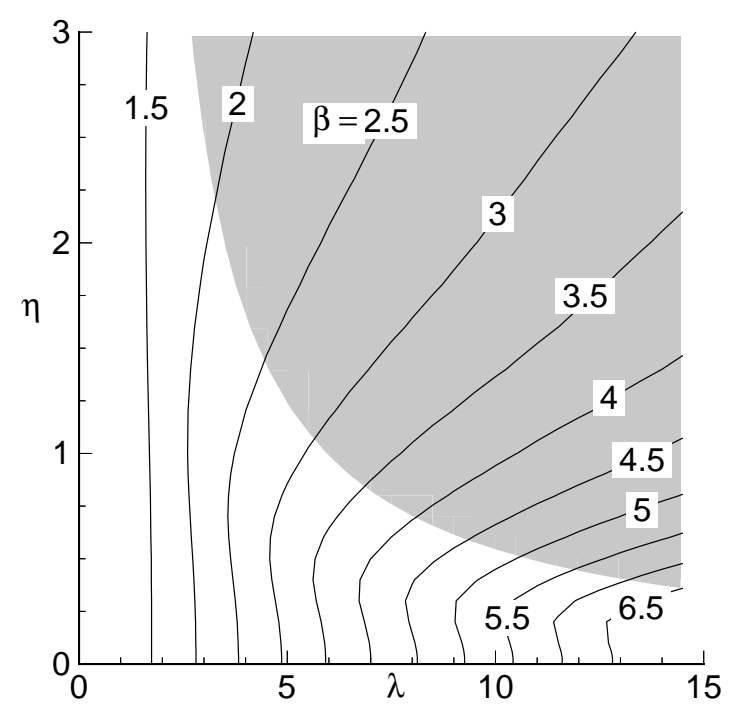

(a) $\chi=0.0$

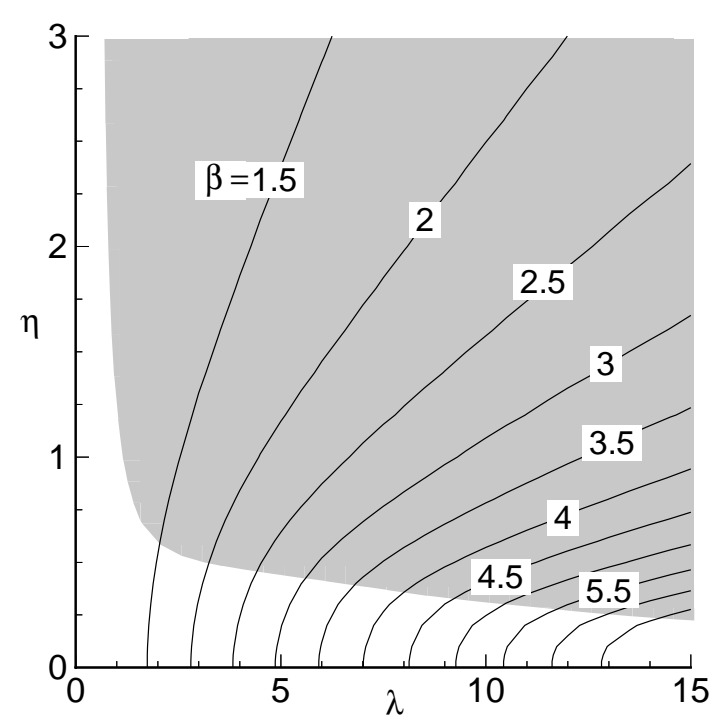

(c) $\chi=1.5$

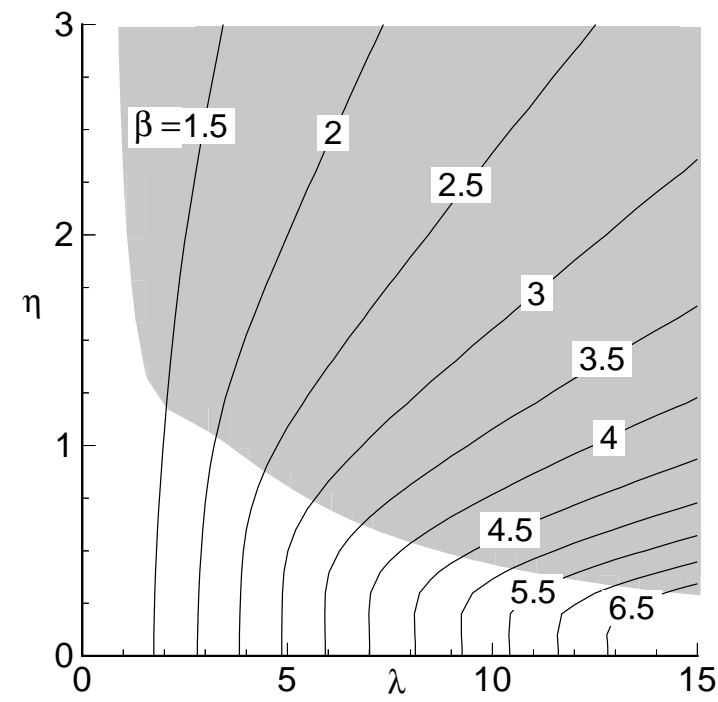

(b) $\chi=0.5$

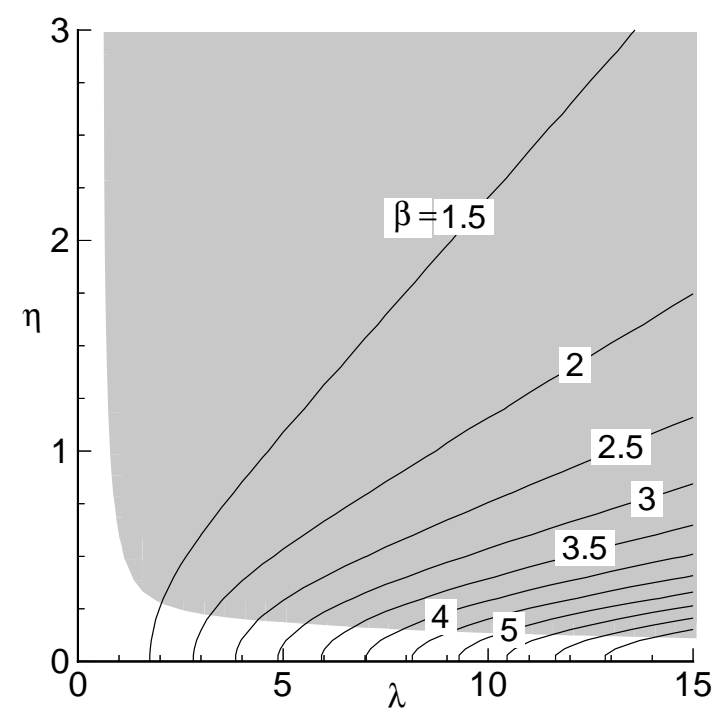

(d) $\chi=6.0$

Figure 6. Contour plot of the bulging factor for a longitudinal crack, $\beta^{L}$, from STAGS analyses, and the linear (unshaded) region of the bulging factor response, as a function of the shell curvature parameter, $\lambda$, and the pressure loading parameter, $\eta$, for several values of the biaxial loading parameter, $\chi$.

bulging factor and the ratio $\lambda / \eta$ is first established for each value of $\chi$. Then, the variation in this relationship is described as a function of $\chi$.

The contour plots of the bulging factor in Fig. 6, and similar contour plots for $\chi=0.25,1.0$, and 3.0, are used to establish the relationship between the nonlinear bulging factor and the ratio $\lambda / \eta$. Along the top and right sides of each contour plot, the values of the nonlinear bulging factors and the corresponding $\lambda$ and $\eta$ values are extracted. The nonlinear bulging factors are plotted for each value of $\chi$ as a function of $\lambda / \eta$ in Fig. 7. Each curve in Fig. 7 is then fitted to a curve to obtain an expression for $\beta_{n l}^{L}$ as a function of $\lambda / \eta$. Through an iterative process, it was determined that the curves in Fig. 7 can be closely approximated by functions of the form

$$
\beta_{n l}^{L}=\sqrt{1+\left[c_{1}(\chi)\right][\lambda / \eta]^{c_{2}(\chi)}}
$$

where the coefficients $c_{1}(\chi)$ and $c_{2}(\chi)$ are determined by curve fitting the results for each value of $\chi$. The coefficients that were obtained from the curve fits of the results in Fig. 7 are given in Table 2. 


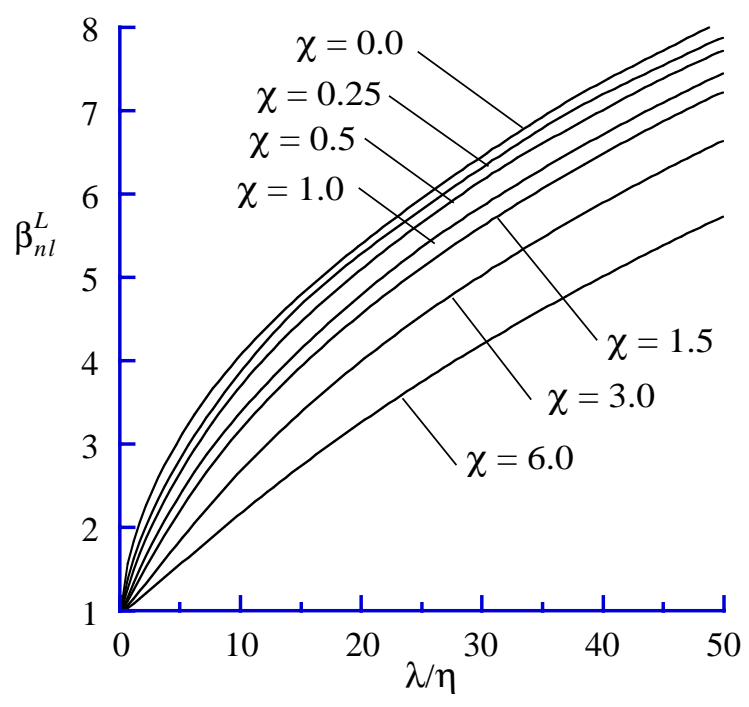

Figure 7. Nonlinear bulging factor for a longitudinal crack, $\beta_{n l}^{L}$ for $\chi=0.0,0.25,0.5,1.0,1.5$, 3.0 , and 6.0 , as a function of the ratio $\lambda / \eta$.

Table 2. Coefficients in Eq. (10) for curve fits of results in Fig. 7

\begin{tabular}{ccc}
\hline \hline$\chi$ & $c_{1}(\chi)$ & $c_{2}(\chi)$ \\
\hline 0.00 & 2.24 & 0.85 \\
0.25 & 1.60 & 0.94 \\
0.50 & 1.32 & 0.98 \\
1.0 & 0.92 & 1.05 \\
1.5 & 0.67 & 1.12 \\
3.0 & 0.33 & 1.27 \\
6.0 & 0.16 & 1.36 \\
\hline \hline
\end{tabular}

The values of the coefficients $c_{1}(\chi)$ and $c_{2}(\chi)$ are plotted as a function of $\chi$ in Fig. 8. In Fig. 8, the solid symbols and the ordinate axis on the left side of the figure reflect the values of the coefficient $c_{1}(\chi)$, and the open symbols and the ordinate axis on the right side of the figure reflect the values of the coefficient $c_{2}(\chi)$. Through an iterative process, it was determined that the data points in Fig. 8 can be closely approximated by the following functions:

$$
c_{1}(\chi)=0.15+1.75 e^{-0.8 \chi}
$$

which is shown in Fig. 8 as a solid line, and

$$
c_{2}(\chi)=1.4-0.52 e^{-0.43 \chi}
$$

which is shown in Fig. 8 as a dashed line. The functions in Eqs. (11) and (12) closely approximate the data in Fig. 8 except for the data for $\chi=0$. At relatively large

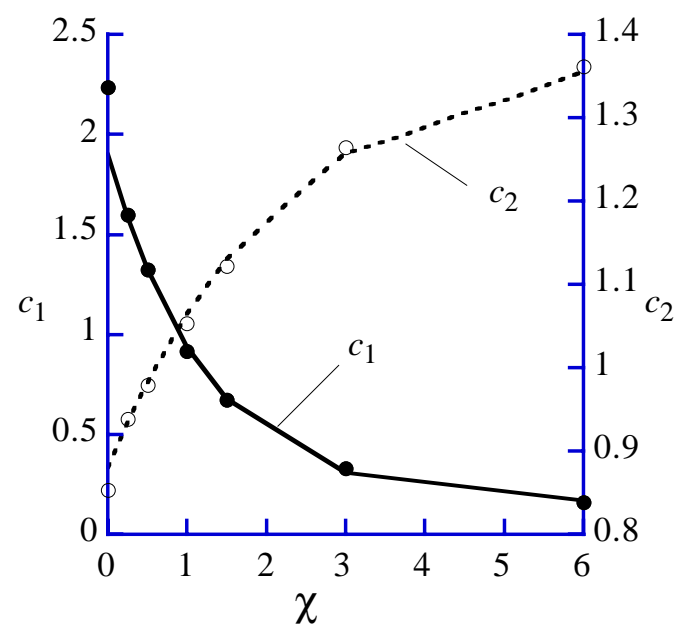

Figure 8. Variation of the coefficients $c_{1}$ and $c_{2}$ as a function of the biaxial loading ratio, $\chi$.

values of the pressure loading parameter, $\eta$, the local shell response for the case where $\chi=0$ is different from the local shell response for cases where the biaxial loading parameter is greater than zero. In the $\chi=0$ case, axial compressive membrane stresses develop adjacent to the crack to equilibrate the axial tensile membrane stresses that develop along the crack edge when the crack bulges. These compressive stresses eventually become large enough to cause the shell to wrinkle near the crack, as shown in Fig. 9. After wrinkling, the value of the bulging factor is not uniquely related to the slope of the radial line, $\lambda / \eta$. This behavior is demonstrated in Fig. $6 \mathrm{a}$ by the divergence of the contour lines from radial lines at large values of $\eta$. For larger values of $\chi$, the compressive stresses near the crack never get large enough to wrinkle the shell, and the value of the bulging factor is uniquely related to the slope of the radial line, $\lambda / \eta$, throughout the nonlinear region of the response.

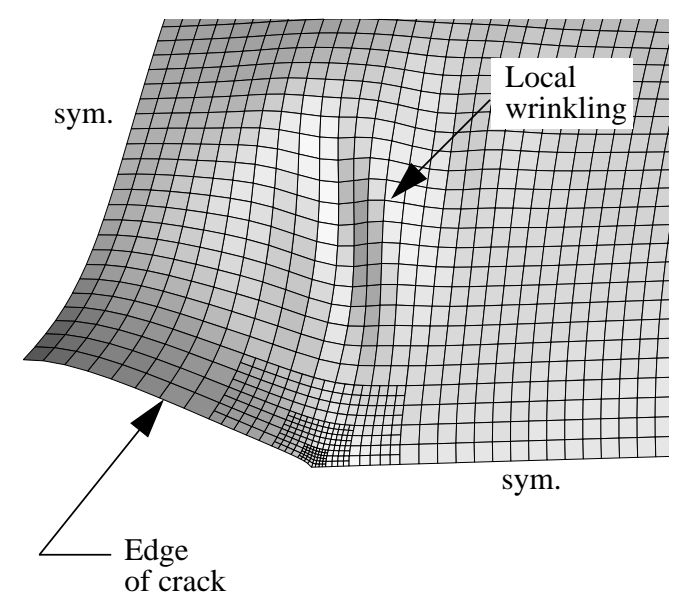

Figure 9. Local shell wrinkling that occurs at high values of load when the biaxial loading parameter, $\chi$, is equal to zero. 
By using Eqs. (11) and (12) with Eq. (10), the nonlinear bulging factor for a longitudinal crack in a cylindrical shell, $\beta_{n l}^{L}$, is expressed in terms of the shell curvature parameter, the pressure loading parameter, and the biaxial loading parameter. The expression for $\beta_{n l}^{L}$ in Eq. (10) will overpredict the bulging factor in the linear region of the response, while the expression for $\beta_{\text {lin }}^{L}$ in Eq. (9) will overpredict the bulging factor in the nonlinear region of the response. The bulging factor over the entire linear and nonlinear regions of the response, for any value of $\lambda, \eta$ and $\chi$, is approximated by taking the minimum of the linear bulging factor estimated by Eq. (9), and the nonlinear bulging factor, estimated by Eq. (10).

$$
\beta_{\text {approx }}^{L}=\min \left(\beta_{\text {lin }}^{L}, \beta_{n l}^{L}\right)
$$

The accuracy of Eq. (13) in representing the bulging factors from the STAGS analyses is demonstrated by the contour plots of the bulging factors for $\chi=0.0$ and 6.0 shown in Figs. 10a and 10b, respectively. In Fig. 10, the bulging factors from the STAGS analyses are shown as solid lines, and the estimates from Eq. (13) are shown as dashed lines. The largest discrepancies between Eq. (13) and the STAGS analyses occur in the transition region between the linear and the nonlinear regions of the response. For $\chi=0$, the discrepancies are less than $10 \%$ over the entire area. For $\chi=6$, the shaded area in the contour plot indicates the region where the discrepancies are greater than $10 \%$. The shaded area is small, and the worst case situation for Eq. (13) overpredicts the bulging factor by $22 \%$.

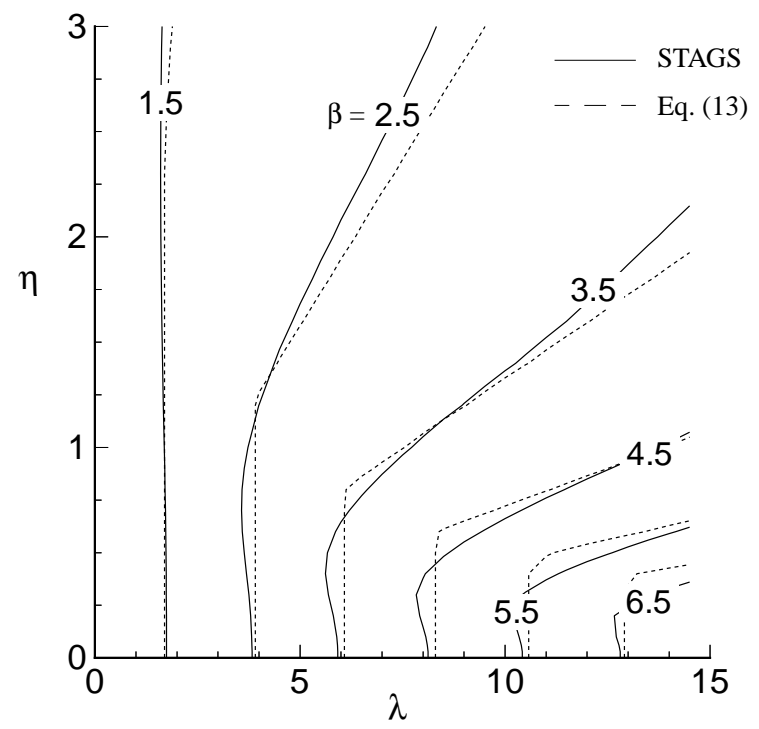

(a) $\chi=0.0$
Circumferential Crack. It was demonstrated previously that the bulging factor for cylindrical shells with circumferential cracks, $\beta^{C}$, has a mild dependence on the biaxial loading parameter. The bulging factors for $\chi=$ 0.5 are a good estimate for $0.25<\chi<6$, and the largest discrepancies are conservative. Thus, the circumferential bulging factor will be characterized for $\chi=0.5$. The bulging factor results from the STAGS analyses of cylindrical shells with circumferential cracks, $\beta^{C}$, are presented as a function of the shell curvature parameter, $\lambda$, and the pressure loading parameter, $\eta$, in Fig. 11. The solid lines in the figure are contour lines, or lines through points with a common value of the bulging factor. The results in the contour plot indicate that the bulging factors monotonically increase with increasing values of $\lambda$, and monotonically decrease with increasing values of $\eta$. This behavior is consistent with the results shown in Fig. 5. The contour plot of the bulging factors for the circumferential crack with $\chi=0.5$ in Fig. 11 is similar to the contour plot of the bulging factors for the longitudinal crack with $\chi=0.5$ shown previously in Fig. $6(\mathrm{~b})$. The primary differences between the bulging factor contour plots for the two crack orientations are that the bulging factor for the circumferential crack is smaller in amplitude, and the contour lines are concentrated nearer to the abscissa of the plot. The bulging factor for very small values of $\eta$, i.e., for locations near the $\lambda$-axis, is exactly the linear response shown previously in Fig. $3 b$. The contour lines are perpendicular to the $\lambda$-axis for very small loads, but the contours bend to the right almost immediately as $\eta$ is increased, and asymptotically approach

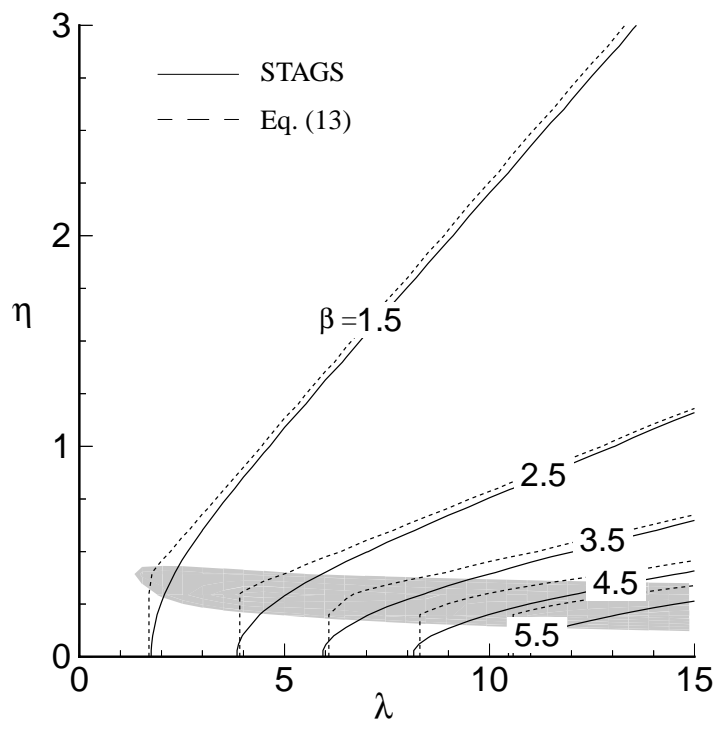

(b) $\chi=6.0$

Figure 10. Contour plot showing the bulging factors for a longitudinal crack, $\beta^{L}$, as computed using STAGS and approximated by Eq. (13), as a function of the shell curvature parameter, $\lambda$, and the loading parameter, $\eta$. 


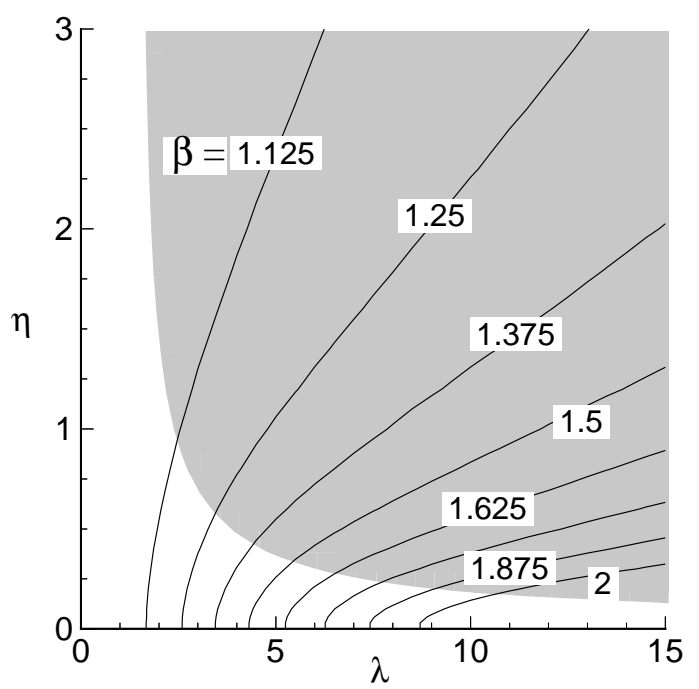

Figure 11. Contour plot of the bulging factor for a circumferential crack, $\beta^{C}$, from STAGS analyses, and the linear (unshaded) region of the bulging factor response, as a function of the shell curvature parameter, $\lambda$, and the pressure loading parameter, $\eta$.

lines which extend radially from the origin. The shaded area of the contour plot in Fig. 11 signifies the nonlinear region of the response where the difference between $\beta^{C}$ and $\beta_{\text {lin }}^{C}$ is greater than $10 \%$. This shaded region is closer to the $\lambda$-axis than it was for the longitudinal crack with $\chi=0.5$, indicating that the transition from the linear bending response to the nonlinear membrane response occurs at smaller values of load for a shell with a circumferential crack than for a shell with a longitudinal crack. The largest differences between $\beta^{C}$ and $\beta_{\text {lin }}^{C}$ occur when $\lambda$ and $\eta$ are both large, which results in $\beta_{\text {lin }}{ }^{C}$ overpredicting $\beta^{C}$ by $100 \%$.

A simple expression, that can be easily used in a design environment, for representing the bulging factor behavior shown in Fig. 11 is obtained by characterizing the linear and nonlinear regions of the response separately. The linear bulging factor for a circumferential crack, $\beta_{\text {lin }}^{C}$, shown previously in Fig. $3 b$, is expressed as a function of $\lambda$ by applying a polynomial curve fit to the numerical data, which gives

$$
\begin{aligned}
\left(\beta_{\text {lin }}^{C}\right)^{2}= & 0.955+0.110 \lambda+0.0637 \lambda^{2} \\
& -0.00534 \lambda^{3}+0.000144 \lambda^{4}
\end{aligned}
$$

An expression for estimating the bulging factor in the nonlinear region of the response, $\beta_{n l}{ }^{C}$, is obtained by utilizing the fact that the contour lines of the bulging factor asymptotically approach radial lines through the origin, and the value of the bulging factor can be uniquely related to the slope of the radial line, i.e., $\lambda / \eta$, which is

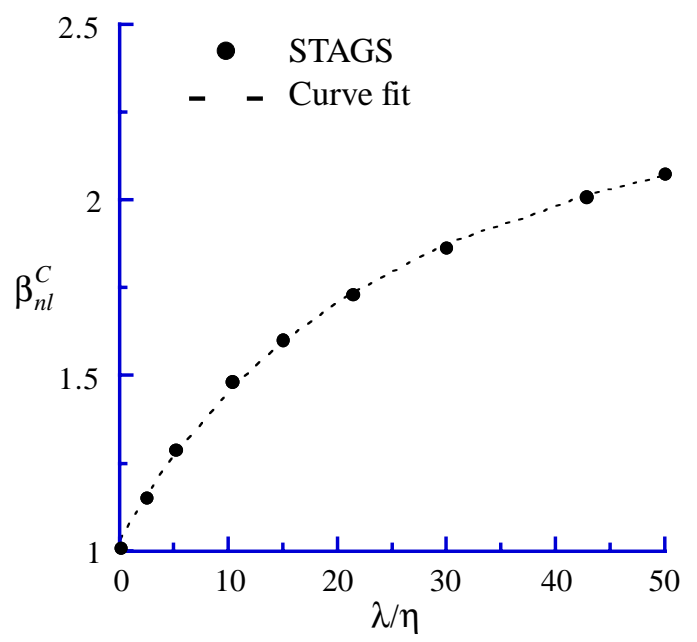

Figure 12. Empirical curve fit to STAGS nonlinear bulging factor for a circumferential crack, $\beta_{n l}^{C}$, as a function of remote values of $\lambda / \eta$.

approached by the contour line asymptotically. The nonlinear bulging factors from the top $(\eta=3)$ and right $(\lambda=15)$ sides of Fig. 11 are plotted as function of $\lambda / \eta$ in Fig. 12, and then expressed as a function of $\lambda / \eta$ by applying a polynomial curve fit to the numerical data, which gives

$$
\begin{aligned}
\left(\beta_{n l}^{C}\right)^{2}=1.05+0.122\left(\frac{\lambda}{\eta}\right) & -0.00161\left(\frac{\lambda}{\eta}\right)^{2} \\
& +9.27 E-6\left(\frac{\lambda}{\eta}\right)^{3}
\end{aligned}
$$

The expression for $\beta_{n l}^{C}$ in Eq. (15) will overpredict the bulging factor in the linear region of the response, while the expression for $\beta_{\text {lin }}^{L}$ in Eq. (14) will overpredict the bulging factor in the nonlinear region of the response. The bulging factor over the entire linear and nonlinear regions of the response, for any value of $\lambda$, $\eta$ and $\chi$, is approximated by taking the minimum of the linear bulging factor estimated by Eq. (14), and the nonlinear bulging factor, estimated by Eq. (15).

$$
\beta_{\text {approx }}^{C}=\min \left(\beta_{\text {lin }}^{C}, \beta_{n l}^{C}\right)
$$

The bulging factors obtained by applying Eq. (16) are compared to the bulging factors from the STAGS analyses in Fig. 13. The bulging factors from the STAGS analyses are shown in Fig. 13 as solid lines, and the estimates from Eq. (16) are shown as dashed lines. The largest discrepancies between Eq. (16) and the STAGS analyses occur in the transition region between the linear and the nonlinear regions of the response. The worst case situation for Eq. (16) overpredicts the bulging factor by $9 \%$. 


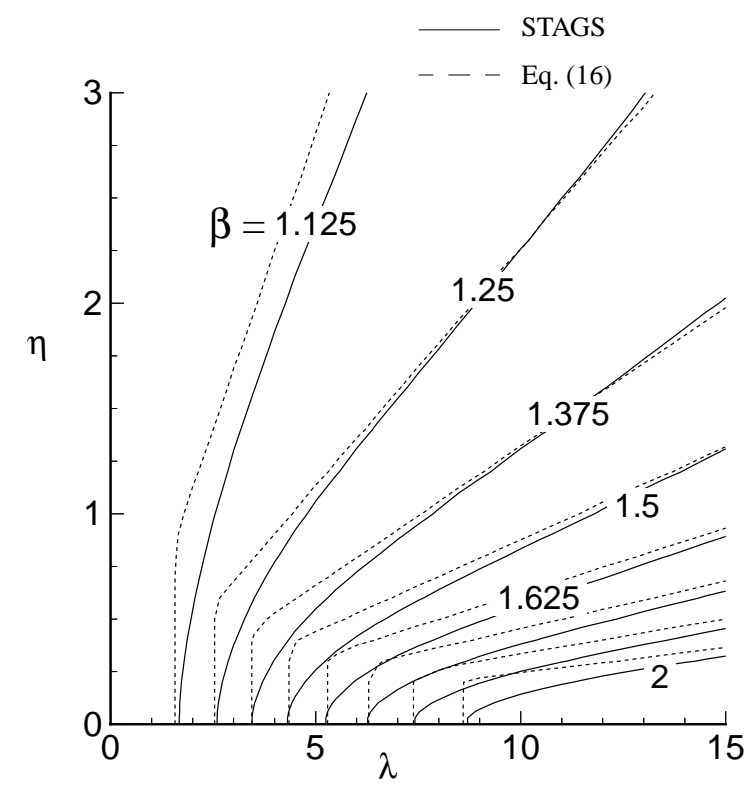

Figure 13. Contour plot showing the bulging factors for a circumferential crack from the STAGS analysis, and from Eq. (16), as a function of the shell curvature parameter, $\lambda$, and the pressure loading parameter, $\eta$.

\section{Concluding Remarks}

The results of a geometrically nonlinear parametric study to determine the effects of shell geometry, and the magnitudes of internal pressure and axial loading on the stress intensity factors at the tips of longitudinal and circumferential cracks in thin unstiffened shells have been presented. The results are normalized by the stress intensity factor for flat plates, and presented in terms of the socalled crack "bulging factor" commonly used in design to represent the effects of shell curvature on the stress intensity factor. The results of the study are presented in terms of three nondimensional parameters: the shell curvature parameter, $\lambda$, which depends on the specific shell geometry; the loading parameter, $\eta$, which depends on the magnitude of the applied internal pressure, the shell radius, and the material stiffness; and the biaxial loading parameter, $\chi$, which is the ratio of the farfield axial stress to the farfield circumferential stress. The shell curvature parameter and loading parameter were suggested by $\mathrm{Bu}-$ diman and Lagace to be the nondimensional parameters for characterizing the nonlinear response of longitudinally and circumferentially cracked cylindrical shells subjected to internal pressure loading.

The nonlinear finite element results of the present study showed that $\lambda, \eta$, and $\chi$ can be used to characterize the nonlinear response of cylindrical shells with longitudinal cracks and subjected to combined internal pressure and axial loads. Furthermore, the results of the present study indicate that the geometrically nonlinear response of a cylindrical shell with a circumferential crack and subjected to combined internal pressure and axial loads is only mildly dependent on the biaxial loading parameter, and thus, can be adequately characterized by the two nondimensional parameters, $\lambda$ and $\eta$. The results also show that the magnitude of the bulging factor is affected by the shell geometry and the shell loading condition, and, that for many shell geometries and load magnitudes, the bulging factor is strongly influenced by the geometrically nonlinear response of a pressurized thin shell. The local response of the shell in the neighborhood of the crack is dominated by linear bending or nonlinear membrane response characteristics, depending on the values of the shell radius, the shell thickness, the crack length, and the magnitudes of the applied internal pressure and axial loads. When the response is dominated by linear bending behavior, the bulging factor response is independent of the loading parameters, and is adequately characterized by expressions that depend only on the shell curvature parameter, $\lambda$. When the response is dominated by nonlinear membrane behavior, the linear bulging factors give extremely conservative predictions of the bulging response. For shells with longitudinal cracks, increasing the biaxial loading parameter promotes the development of nonlinear membrane behavior near the crack, but decreasing the biaxial loading parameter delays the development of nonlinear membrane behavior and extends the region of the response which is dominated by linear bending. Simple empirical expressions for the bulging factor are derived from the numerical results and are shown to predict accurately the linear and nonlinear response of shells with longitudinal and circumferential cracks.

\section{$\underline{\text { References }}$}

${ }^{1}$ Folias, E. S., "An Axial Crack in a Pressurized Cylindrical Shell," International Journal of Fracture Mechanics, Vol. 1, No. 2, 1965, pp. 104-113.

${ }^{2}$ Folias, E. S., "A Circumferential Crack in a Pressurized Cylindrical Shell," International Journal of Fracture Mechanics, Vol. 3, 1967, pp. 1-12.

${ }^{3}$ Folias, E. S., "On the Effect of Initial Curvature on Cracked Flat Sheets," International Journal of Fracture Mechanics, Vol. 5, No. 4, December 1969, pp. 327-346.

${ }^{4}$ Folias, E. S., "Asymptotic Approximations to Crack Problems in Shells," Mechanics of Fracture - Plates and Shells with Cracks, G. C. Sih, H. C. van Elst, and D. Broek, eds., Noordhoff International, Leyden, 1977, pp. 117-160.

${ }^{5}$ Copely, L. G., and Sanders, J. L., Jr., “A Longitudinal Crack in a Cylindrical Shell under Internal Pressure," International Journal of Fracture Mechanics, Vol. 5, No. 2, June 1969, pp. 117-131. 
${ }^{6}$ Erdogan, F., and Kibler, J. J., "Cylindrical and Spherical Shells with Cracks," International Journal of Fracture Mechanics, Vol. 5, No. 3, September 1969, pp. 229-237.

${ }^{7}$ Erdogan, F., and Ratwani, M., "Fatigue and Fracture of Cylindrical Shells Containing a Circumferential Crack," International Journal of Fracture Mechanics, Vol. 6, No. 4, September 1970, pp. 379-392.

${ }^{8}$ Duncan-Fama, M. E., and Sanders, J. L., Jr., "A Circumferential Crack in a Cylindrical Shell under Tension," International Journal of Fracture Mechanics, Vol. 8, No. 1, March 1972, pp. 15-20.

${ }^{9}$ Peters, Roger W., and Kuhn, Paul, "Bursting Strength of Unstiffened Pressure Cylinders with Slits," NACA TN 3993, April 1957.

${ }^{10}$ Anderson, Robert B., and Sullivan, Timothy L., "Fracture Mechanics of Through-Cracked Cylindrical Pressure Vessels," NASA TN D-3252, February 1966.

${ }^{11}$ Chen, D., "Bulging of Fatigue Cracks in a Pressurized Aircraft Fuselage," Ph.D. Thesis, Delft University of Technology, Delft, The Netherlands, Report LR-647, October 1990.

${ }^{12}$ Jeong, D. Y., and Tong, P., "Nonlinear Bulging Factor Based on R-Curve Data," Proceedings of the FAA/ NASA International Symposium on Advanced Structural Integrity Methods for Airframe Durability and Damage Tolerance, September 1994, pp. 327-338.

${ }^{13}$ Bakuckas, J. G., Ngugen, P. V., and Bigelow, C. A., "Engineering Fracture Parameters for Bulging Cracks in Pressurized Unstiffened Curved Panels," Proceedings of the FAA-NASA Symposium on Continued Airworthiness of Aircraft Structures, DOD/FAA/AR-97/2, 1996.

${ }^{14}$ Bakuckas, J. G., Jr., Nguyen, P. V., Bigelow, C. A., and Broek, D., "Bulging Factors for Predicting Residual Strength of Fuselage Panels," Presented at the International Conference on Aeronautical Fatigue, Edinburgh, Scotland, June 18-20, 1997.

${ }^{15}$ Riks, E., Brogan, F. A., and Rankin, C. C., "Bulging Cracks in Pressurized Fuselages: A Procedure for Computation," in Analytical and Computational Models of Shells, Noor, A. K., Belytschko, T., and Simo, J. C., eds., The American Society of Mechanical Engineers, ASMECED, Vol. 3, 1989.

${ }^{16}$ Budiman, H. T., and Lagace, P. A., "Nondimensional Parameters for Geometric Nonlinear Effects in Pressurized Cylinders with Axial Cracks," Journal of Applied Mechanics, Vol. 64, 1997, pp. 401-407.

${ }^{17}$ Budiman, H. T., "Mechanisms of Damage Tolerance and Arrest in Pressurized Composite Cylinders," Ph.D. Thesis, Department of Aeronautics and Astronautics, Massachusetts Institute of Technology, Cambridge, MA, 1996.
${ }^{18}$ Yamaki, N., Elastic Stability of Circular Cylindrical Shells, North-Holland Series in Applied Mathematics and Mechanics, Vol. 27, E. Becker, B. Budiansky, W. T. Koiter, and H. A. Lauwerier, eds., North Holland, Amsterdam, 1984.

${ }^{19}$ Rankin, C. C., Brogan, F. A., Loden, W. A., and Cabiness, H. D., "STAGS User Manual, Version 3.0," Lockheed Martin Missiles \& Space Co., Inc., Rept. LMSC P032594, March 1999.

${ }^{20}$ Young, R. D., Rose, C. A., and Starnes, J. H., Jr., "Nonlinear Local Bending Response and Bulging Factors for Longitudinal and Circumferential Cracks in Pressurized Shells," Proceedings of the 3rd Joint FAA/DoD/ NASA Conference on Aging Aircraft, Albuquerque, NM, September 20-23, 1999.

${ }^{21}$ Rose, C. A., Young, R. D., and Starnes, J. H., Jr., "Nonlinear Local Bending Response and Bulging Factors for Longitudinal Cracks in Pressurized Cylindrical Shells," Presented at the 40th AIAA/ASME/ASCE/AHS/ ASC Structures, Structural Dynamics, and Materials Conference, St. Louis, MO, April 12-15, 1999, AIAA Paper No. 99-1412.

${ }^{22}$ Broek, D., Elementary Engineering Fracture Mechanics, Sijthoff \& Noordhoff, 1978.

${ }^{23}$ Riks, E., "Bulging Cracks in Pressurized Fuselages: A Numerical Study," NLR Report NLR-MP-87058 U, NLR National Aerospace Laboratory, The Netherlands, 1978.

${ }^{24}$ Potyondy, D. O., Wawrzynek, P., A., and Ingraffea, A. R., "Discrete Crack Growth Analysis Methodology for Through Cracks in Pressurized Fuselage Structures," International Journal for Numerical Methods in Engineering, Vol. 38, 1995, pp. 1611-1633.

${ }^{25}$ Rankin, C. C., and Brogan, F. A., "The Computational Structural Mechanics Testbed Structural Element Processor ES5: STAGS Shell Element," NASA Contractor Report 4358, May 1991.

${ }^{26}$ Riks, E., "Some Computational Aspects of the Stability Analysis of Nonlinear Structures," Computational Methods in Applied Mechanics and Engineering, Vol. 47, 1984, pp. 219-259.

${ }^{27}$ Riks, E., "Progress in Collapse Analysis," Journal of Pressure Vessel Technology, Vol. 109, February 1987, pp. 27-41.

${ }^{28}$ Rybicki, E. F., and Kanninen, M. F., "A Finite Element Calculation of Stress Intensity Factors by a Modified Crack Closure Integral," Engineering Fracture Mechanics, Vol. 9, 1977, pp. 931-938. 